\title{
From Separation to Incorporation - A Full-Circle Application of Computational Approaches to Performance-Based Architectural Design
}

\author{
Yuhan Chen, Youyu Lu, Tianyi Gu, Zhirui Bian, Likai Wang ${ }^{(凶)}$, and Ziyu Tong \\ Nanjing University, Hankou Road 22, Nanjing, Jiangsu, China \\ wang.likai@nju.edu.cn
}

\begin{abstract}
In performance-based architectural design, most existing techniques and design approaches to assisting designers are primarily for a single design problem such as building massing, spatial layouts, or facade design. However, architectural design is a synthesis process that considers multiple design problems. Thus, for achieving an overall improvement in building performance, it is critical to incorporate computational techniques and methods into all key design problems. In this regard, this paper presents a full-circle application of different computational design approaches and tools to exploit the potential of building performance in driving architectural design towards more novel and sustainable buildings as well as to explore new research design paradigms for performance-based architectural design in real-world design scenarios. This paper takes a commercial complex building design as an example to demonstrate how building performance can be incorporated into different building design problems and reflect on the limitations of existing tools in supporting the architectural design.
\end{abstract}

Keywords: Performance-based architectural design - Computational design · Building performance $\cdot$ Design optimization $\cdot$ Research design

\section{Introduction}

Performance-based design has become a trend in architecture and has been widely applied to building massing, floor plan layouts, and façade design. When confronting the complex challenge of designing a high-performance building, computational design approaches and tools are becoming an indispensable component in achieving a performance-based architectural design. Over the past decade, there have been a fastgrowing number of design tools and methods proposed by researchers and developers, such as Galapagos (Rutten 2013) and Octopus (Vierlinger 2013) to offer architects assists in the building design process. However, the applications or studies of using these tools are often too targeted: they typically focus on one specific design problem, such as massing generation (Wang et al. 2020a), floor plan layouts (Dino 2016), or 
facade design (Wright et al. 2014). While the relevant studies show that using computational optimization or other computational techniques such as simulations can help to improve the performance of the building design, there are few attempts at or investigation on incorporating these computational techniques into real-world architectural design scenarios and reflecting on the gap between research and practice.

Since architectural design is a process that needs to synergize different design problems, the exploration of using different computational design tools, from beginning to end, in all aspects and elements of architectural design is essential to make performance a real driving factor consistent in a whole architectural design circle. In this regard, it is pertinent to examine how to incorporate these existing computational design techniques and approaches in a complete design circle. Hence, this paper presents a full circle application of different computational design approaches based on an undergraduate design studio project, intending to explore a new design paradigm for performance-based architectural design.

In addition, as an initial attempt at combining different computational techniques and approaches in a complete architectural design task, the study bears many limitations from the practical point of view. However, it should be stressed that the contribution of this paper is to demonstrate an example of how computational design techniques and approaches can be applied and promoted a research design (design by research) paradigm in architectural design, where these techniques and approaches serve as a means of systematic inquiry of the design problem, helping the designers overcome data-poor situations, and eventually, synthesizing building performance into the design process. At the same time, while the study does not advance computational design approaches from the technical perspective, the example presented is also aimed to provides an opportunity for the research community to inspect the gap that needs to be filled between research and practice. Thus, in the conclusion of the paper, we discuss the deficiency in the current computational design approaches and techniques that we identified during the presented design process.

\section{Method}

In order to make building performance a driving factor in architectural design, we apply computational design techniques and approaches to different design stages/problems and try to improve the performance using different computational design methods. The design process still follows the ordinary architectural design process, from stages of building massing design, to spatial layouts, and, finally, to facade design. In each design stage, different computational tools are used (Fig. 1). Beyond performance concerns, we also try to incorporate other architectural design intentions such as functions, aesthetics, and building codes or regulations into the computational design.

First, for the building massing design stage, EvoMass (Wang et al. 2020b), a plugin for agile building massing generation and exploration, and DIVA, a radiance-based performance simulation tool in the Rhino-Grasshopper, are used. Unlike typical applications of performance-based design only considering performance factors, the architectural design intentions are also transformed into constraints and objectives for the optimization.

Second, for floor plan design, performance simulation based on DIVA is first carried out to evaluate the indoor environment quality in the different sections of the building. On 


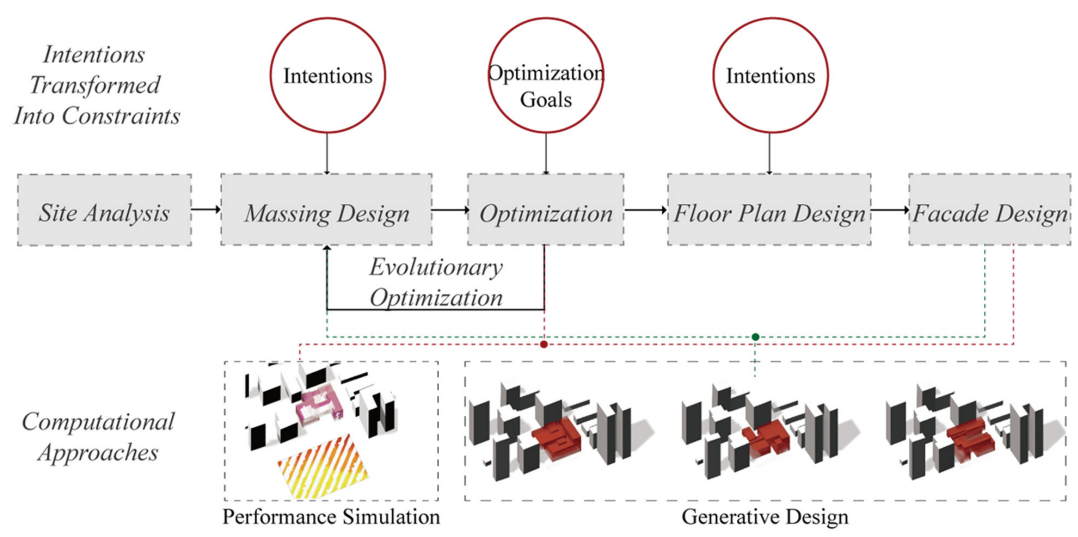

Fig. 1. The proposed design process

this basis, different functional spaces, such as exhibition and office rooms, are arranged primarily according to the environmental quality requirements of these spaces.

Finally, the building's facade design is generated by a combination of methods of generative design and performance simulation to achieve an adaptive building skin that can well respond to the surrounding urban environment as well as the local climate condition.

\section{Case Study}

To illustrate the efficacy of the proposed design process, a case-study design of a commercial complex building in Shanghai is presented to elaborate on how different computational tools are used (Fig. 2). Located in Shanghai, the site has a park on its north and is surrounded by several high- and middle-rise buildings on its south, west, and east sides. The building's surrounding urban environment poses a huge challenge to achieve a favorable performance in the design. Thus, the primary objective of the case study focuses on the daylighting performance of the building and its impact on an adjacent public park. In the following part of this section, the process of our application utilizing multiple computational tools to each of the design stages/problems that lead to the final design work will be introduced.

\subsection{Building Massing Design}

At the outset of building massing design, design intentions are specified to define the overall properties of the building massing and then guide the process of optimization search for satisficing solutions. The properties include the range of its overall size and maximal gross floor area. These intentions consider the site and the program (Fig. 3): First, the building massing design that will be transformed into an office complex building which needs to meet the basic functional requirements since we conceive the final design as a practical project. Second, the performance of the building is expected to be 


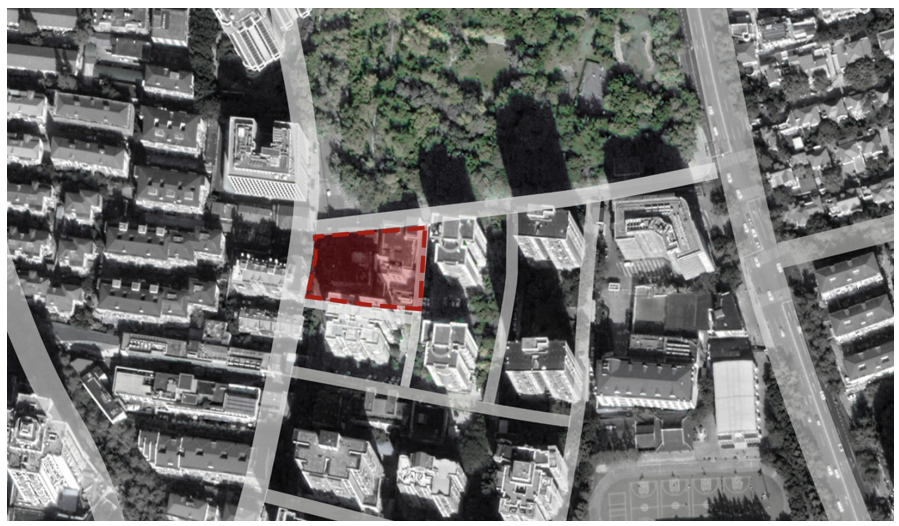

Fig. 2. Site overview

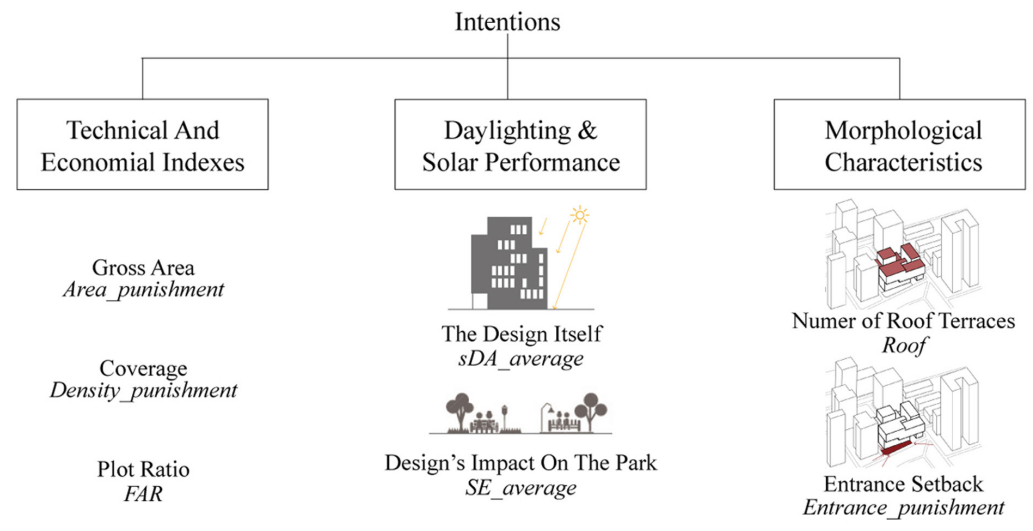

Fig. 3. Intentions in relation to the settings for optimization

maximized, and its negative impact on the park is to be minimized. Third, other requirements and intentions related to the formal characteristics of the building need are also considered.

In the building massing design optimization process, functional requirements, performance requirements, and formal characteristics are all transformed into quantifiable indicators that can be encapsulated into the fitness function.

For functional requirements, although building performance is our primary design goal, functional requirements are still the most important concern in architectural design. In this case study, the functional indicators evaluating against each of the generated building massing designs including total area, building density, floor area ratio (FAR), and the number of floors (Area: $15000 \mathrm{~m}^{2}$, Density: 0.5, FAR: 5, Number of Floors: 8).

For performance requirements, due to the building plot situated in a complex urban environment, this study focuses on the building massing design's performance on daylighting and thermal comfort quality of interior space of the generated building design 
and its impact on the park. The evaluations were carried out based on the DIVA simulation tool in the grasshopper platform. We took the average value of annual natural lighting and the average solar heat radiation value of the park as the two fitness-related values affecting the generation and optimization of the building massing and intend that the generated volume can have higher overall fitness.

For formal characteristics, we determined that as urban architecture and a public building, the design should be spatially and formally interesting, friendly for use, and open to urban space. Considering that the building is faced with the main road on its entrance, we hope that the entrance of the building can retreat and form a semi-outdoor public space for pedestrians. In addition, we hope that the volume of the building will display richness in shape so that we have more chances to provide shared and activity spaces.

Note that, in a similar way to ordinary architectural design, our design process was also an iteratively reflective process (Schön 1992), where we found unintended consequences from optimization results and, thereby, added up new intentions into the optimization. In other words, these intentions were iteratively included in the design process alongside using optimization, and it took us several iterations to reflect on the on-progress optimization result, reformulate our optimization design problem (fitness functions), and gradually reach the final design.

In the optimization stage, all the above-mentioned fitness-related values are used for optimization. The final fitness function consists of multiple variables and can be expressed as:

$$
\text { Fitness }=S I * s D A * n u m \_r o o f * p \_ \text {area } * p \_d e n * p \_e n t r a n c e * n u m \_r o o f
$$

Among these parameters, $S I$ and $S D A$ respectively represent the solar irradiation received by the park and the daylighting value of the building, $p \_$area the punished value of the total area, $p \_d e n$ represents the punished value of density, $p \_$entrance represents the punished value of the projected area of entrance, and num_roof represents the number of roofs.

As shown in the fitness function, we use a penalty function to control the result of optimization. If the values of building area, building density, and entrance area exceed the preset values, the penalty function will be used to punish the excess part, resulting in a lower value of its fitness. In this way, the more the values exceed the expected value, the lower the value of the variable in the formula will be. As such, we managed to interfere with the optimization process by controlling the significance of the parameters contributing to the value of fitness.

We used the optimization algorithm embedded in EvoMass, called SSIEA (Wang et al. 2020c), for running the optimization. SSIEA is a diversity-guided algorithm that can produce optimization results with variants showing large design differentiation. The diversity in the optimization, on the one hand, helps us to extract more information from the optimization result and obtain a better understanding of the design problem. On the other hand, the design diversity in the optimization result also provides more optional design solutions that we can choose from when considering other unquantifiable concerns. Figure 4 shows the optimal design variants found by the last iteration of design optimization that can generally satisfy all our intentions and performance concerns, and we selected one design variant for further design development. 

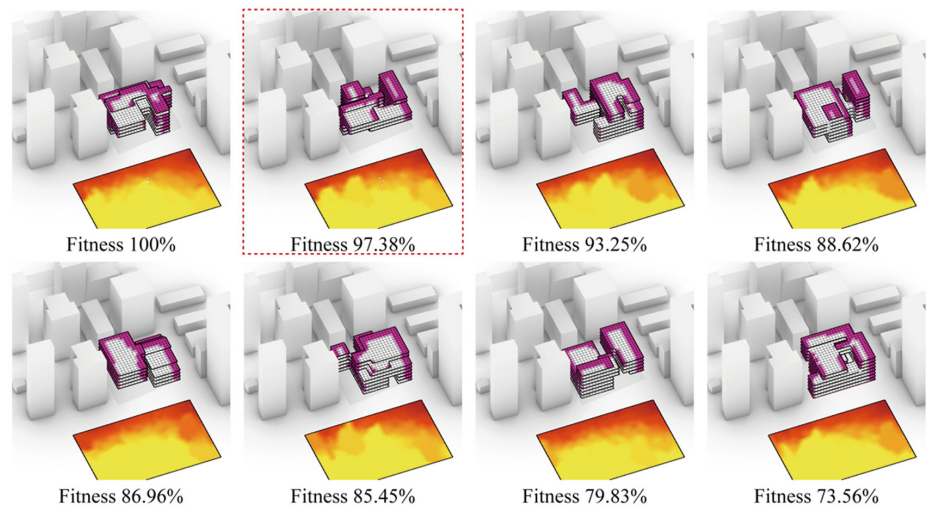

Fig. 4. Final building massing design optimization results (red-dotted rectangle: the selected design)

\subsection{Floor Plan Design}

In conventional floor plan design, the relationship among different functional spaces is the priority, but in this study, we treat performance equally important: we started from the analysis of natural light accessibility simulated by DIVA and allocated each of the functional spaces according to each volume's characteristics.

Based on the design variant selected in the building massing design stage, we first adjusted the volume to make the spatial logic clearer. We found the selected design solution consists of five interlocking blocks: three large volumes enclosing and spinning clockwise, located on the corners of the building plot, one small vertical volume inserted into the former three, and a last horizontal one connecting all the vertical ones.

According to the daylighting simulation results, we arranged the function of each block according to its daylighting quality as well as functional requirements. (1) According to the natural lighting simulation, the small vertical volume has the worst daylighting quality and situates at the intersection of all other blocks. Thus, it is most suitable to serve as the vertical circulation; (2) for the three large volumes, the southern one has better daylighting, therefore used as a shared functional space, (3) the northern one has its lower part directly accessible to the urban street interface, therefore chosen as the cultural space such as book store and coffee shops facing the city, (4) the western one relatively independent with an unfavorable daylighting condition was used as the office area; (5) as for the horizontal volume, the large and unobstructed plan, and northward lighting makes it suitable for the exhibition that requires a coherent and flowing space. In this way, several large functional spaces are sorted out, and then other subordinate spaces and functions such as fire escalators, restrooms, and receptions were placed (Fig. 5). 

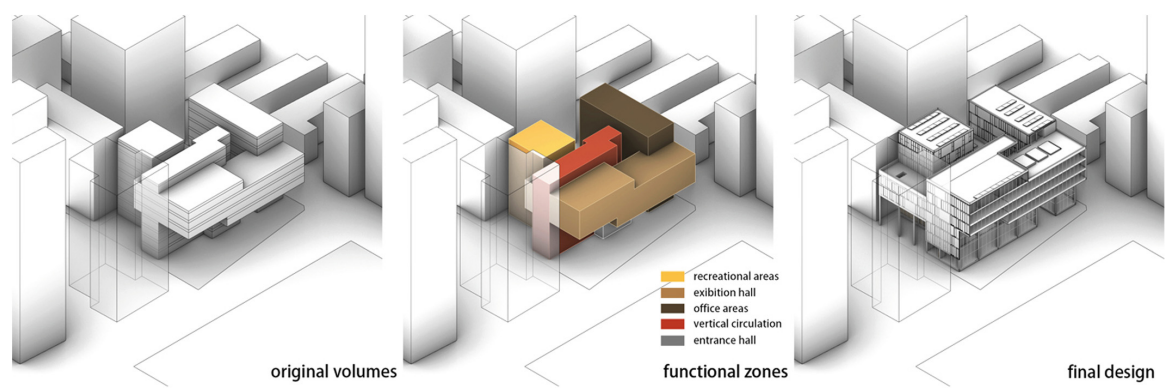

Fig. 5. Development of floor plan design

\subsection{Facade Design}

Facades are an important element in architectural design and play a critical role in indoor thermal comfort and daylighting. Hence, we developed an algorithm that can generate a facade based on the solar irradiation received by the surface of the volume. Based on the solar irradiation intensity, the algorithm calculates the specific window-to-wall ratio of each facade surface, so as to make the facade more responsive and adaptive to the environment (Fig. 6).
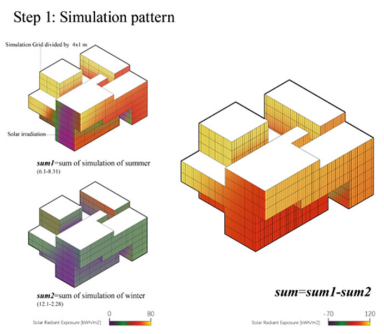

Step 2: Facades division pattern

Step 3: Fenestration pattern
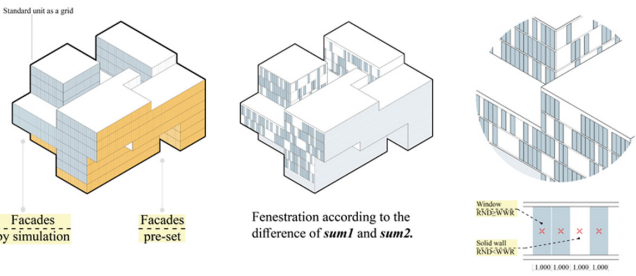

Fig. 6. The generation process of the façade pattern

First, based on the building volume and the interior functions that have been defined so far, each of the facade surfaces is assigned with different types of facade schemes according to the function or thermal requirement of the space. For instance, the threestory cantilevered large space involving assembly functions such as exhibition and viewing, full glazing curtain walls are used. In contrast, the facade of other spaces for commercial and office rooms on the south side of the plan, its relationship with thermal comfort should be considered.

Second, we used the DIVA to calculate the total solar irradiation on each facade surface in summers and winters, and do the difference calculation, visualize the results through an RGB image, and use the color value to express the difference. The darker the part is, the smaller the difference, on the contrary, the brighter, the greater the difference. Because the received solar irradiation in summers is always higher than that in winters, if the difference of heat radiation between summer and winter of the facade surface is 
small, it means that this part of the facade surface is less overheated in summers while can be also heated in winters. Thus, using a large window-to-wall ratio is more favorable. If the heat radiation difference is large, it is not suitable for large window-to-wall ratios, as the window may allow excessive solar irradiation to enter the building in summers while its role in passive heating in winters is trivial.

Finally, with the data controlling the window-to-wall ratio for each of the facade surfaces, we modularized the facade and set each facade unit to $3.7 \times 1.0 \mathrm{~m}$. To avoid repeated facade patterns, we assigned a random number to each facade unit and compare this number to the window-to-wall ratio to decide whether this unit is windows or walls.

\subsection{Final Design Work}

Figure 7 shows the final design work of this case study. The final design work presents many underlying architectural implications related to building performance. For example, in regards to the building massing design, the building shows a strong tendency towards strip shape volumes, which is more favorable for daylighting due to shallow floor plans. In addition, the building massing has a lower northwest corner in the building height that allows more sunlight to reach the park. In regards to the floor plan and facade design, the design of the facade surfaces (fully glazing curtain walls or fenestrations) reflects the function, daylighting, and thermal comfort requirement of the space behind it.

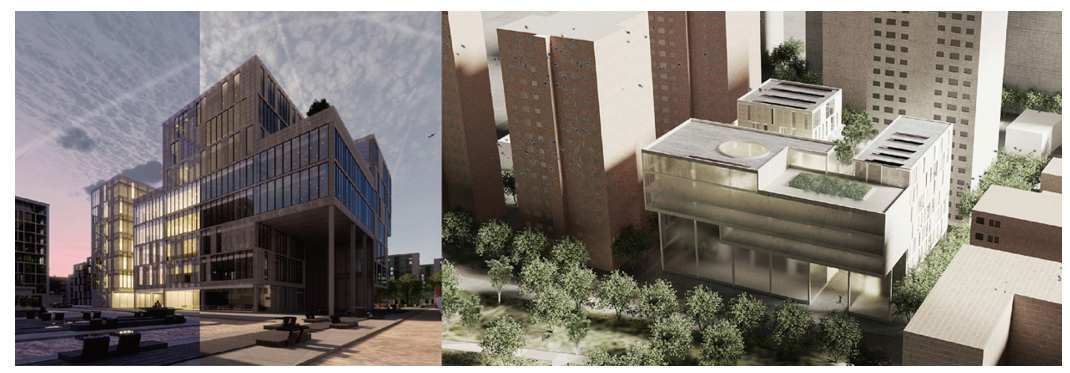

Fig. 7. Artistic impression of the final design work (left: pedestrian perspective, right: bird view)

Other than the performance consideration, the design work also shows architectural features reflecting our design intentions. The multiple building volumes allow for more rooftop terraces that provide space encouraging social events, viewing, and relaxation. The entrance with a large overhanging structure shows a friendly gesture to pedestrians on the streets and in the park and welcomes them to enter the building. The building facade, serving as the interface between the building and urban environment, not only ensures that each section of the building has desirable daylighting and thermal comfort conditions but also satisfies the visual requirements of different functional spaces. For example, higher openness to the exhibition area but more privacy for the office rooms. 


\section{Discussion and Conclusion}

The preceding sections illustrate an example of how different computational design techniques and approaches can be applied to an architectural design task from beginning to end with using building performance as a driving factor. While the example shows the potential in existing techniques and approaches to assist designers in overcoming datapoor situations and achieving a performance-informed, it is more important to reflect on this process and identify the gap between the functionality of these techniques and approaches and the need in design processes.

The most significant issue when we reflect on our example is that the detachment of design objects and design stages. During the design process, the three design objects were designed separately, and the interaction among these objects was ignored, despite its importance, which has been demonstrated recently (Zhang et al. 2021). This, on the one hand, is because the tight design schedule did not allow us to undertake more iterations to bring the new design information found in the later design stages (floor plan and façade design) to the preceding design stage (building massing design). On the other hand, it also lacks applicable approaches that can incorporate these design objects in a integrated design process. Thus, linking these design objects in one design generation process is a possible solution to address this issue. The second issue is the lack of handy design tools. Apart from the building massing design, the other design stages required us to spend a great amount of time and effort to establish design simulation and design generation workflows from scratch, which not only slowed down the design process but also made the design exploration inefficient due to the tedious and repeated workflow establishing process. Thus, transforming the state-of-art into agile and flexible design tools could a crucial step to bridge the gap between research and practice.

To conclude, this paper demonstrates the usage and efficacy of computational design techniques and approaches in performance-based architectural design and promoting a research design process. While the potential of the computational design techniques and approaches in supporting sustainable building design has been clearly proven in this study, we also argued that there is still a large gap between the state-of-the-art and practice. Hence, this study also attempts to an opportunity to inspect and evaluate existing techniques and tools from a designers' perspective as well as to understand what designers are expecting in the future.

\section{References}

1. Dino, I.G.: An evolutionary approach for 3D architectural space layout design exploration. Autom. Constr. 69, 131-150 (2016). https://doi.org/10.1016/j.autcon.2016.05.020

2. Rutten, D.: Galapagos: on the logic and limitations of generic solvers. Archit. Des. 83(2), 132-135 (2013). https://doi.org/10.1002/ad.1568

3. Schön, D.A.: Designing as reflective conversation with the materials of a design situation. Knowl.-Based Syst. 5(1), 3-14 (1992)

4. Vierlinger, R.: Multi objective design interface. University of Applied Arts Vienna (2013)

5. Wang, L., et al.: Algorithmic generation of architectural massing models for building design optimisation parametric modelling using subtractive and additive form generation principles. In: RE: Anthropocene, Proceedings of the 25th International Conference of the Association 
for Computer-Aided Architectural Design Research in Asia (CAADRIA) 2020, pp. 385-394 (2020a)

6. Wang, L., et al.: Enabling optimisation-based exploration for building massing design a codingfree evolutionary building massing design toolkit in rhino-grasshopper. In: RE: Anthropocene, Proceedings of the 25th International Conference of the Association for Computer-Aided Architectural Design Research in Asia (CAADRIA) 2020, pp. 255-264 (2020b)

7. Wang, L., Janssen, P., Ji, G.: SSIEA: a hybrid evolutionary algorithm for supporting conceptual architectural design. Artif. Intell. Eng. Des. Anal. Manuf. 34(4), 458-476 (2020c). https://doi. org/10.1017/S0890060420000281

8. Wright, J.A., et al.: Multi-objective optimization of cellular fenestration by an evolutionary algorithm. J. Build. Perform. Simul. 7(1), 33-51 (2014)

9. Zhang, H., Wang, L., Ji, G.: The Synergy of building massing and facade - an Evo-Devo approach for performance-based design optimization combining facade design with building massing. In: PROJECTIONS - Proceedings of the 26th CAADRIA Conference. The Chinese University of Hong Kong and Online, Hong Kong, pp. 451-460 (2021)

Open Access This chapter is licensed under the terms of the Creative Commons Attribution 4.0 International License (http://creativecommons.org/licenses/by/4.0/), which permits use, sharing, adaptation, distribution and reproduction in any medium or format, as long as you give appropriate credit to the original author(s) and the source, provide a link to the Creative Commons license and indicate if changes were made.

The images or other third party material in this chapter are included in the chapter's Creative Commons license, unless indicated otherwise in a credit line to the material. If material is not included in the chapter's Creative Commons license and your intended use is not permitted by statutory regulation or exceeds the permitted use, you will need to obtain permission directly from the copyright holder.

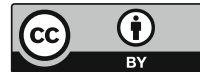

